\title{
Effects of the Inlet Velocity Profiles on the Prediction of Velocity Distribution inside an Electrostatic Precipitator (ESP)
}

\author{
A. F. Yusop, R. Mamat, M. H. Mat Yasin, and S. Suhaimi
}

\begin{abstract}
In this study, the development of CFD model for the description of operation of electrostatic precipitator has been developed. The model is based on the simulation analysis whereby focused on the gas flow by time-averaged conservation equation of mass and momentum. 2D geometry model was extensively applied due to ease the geometry and analysis. The flow simulation was performed by using computational fluid dynamics (CFD) code Fluent. Numerical calculations for the air flow were calculated using the Reynolds-averaged Navier-Stokes equation coupled with the realizable $k-\varepsilon$ turbulence model equations. Simulations were performed using three velocities at the inlet boundary by considering a uniform (ideal) velocity profile. Simulation with the low velocity profile gives a better flow rate efficiency between the outlet and inlet of the ESP. Overall, all the three velocities give more than $90 \%$ of efficiency of the air flow inside ESP chamber.
\end{abstract}

Index Terms-Electrostatic precipitator, CFD, velocity and flow simulation.

\section{INTRODUCTION}

Particle emission can be controlled by using electrostatic precipitator (ESP) and are mostly used in power plant or others process industries. Thus, the flow inside an ESP needs to be aerodynamic in order to have a high efficiency of performance. The performance of ESP is affected by the geometry inside of an ESP. The more complex geometry, the complex flow distribution will be arise. Therefore, the geometry inside an ESP is a crucial part and need considerable attention. Due to the complexity of the geometry inside an ESP, it is difficult to carry out detailed measurement of the fluid flow. In order to perform the analysis, CFD provide a good agreement as an alternative method and less expensive to study the behavior inside the ESP. By choosing a suitable CFD model, it will help to predict the flow characteristic inside the ESP and optimizing the flow distribution based on the proposed modification.

Several methodologies have been studied in the operation of the ESP. One of them is experimental approach which is the study of laboratory scale ESP combined with simplified numerical modeling [1]-[4]. In these researches, most of the parameter such as discharge wire diameter, wire to wire and wire to plate spacing, air flow velocity and applied wire voltage are tested and optimized. Classical theoretical models such as Deutsch and Cooperman were compared to the

Manuscript received February 5, 2013; revised June 22, 2013

This work was supported in part by the Universiti Malaysia Pahang Grant (RDU100334).

The authors are with Universiti Malaysia Pahang, 26600, Pekan, Pahang, Malaysia._(e-mail: fitri43@gmail.com, rizalman@ump.edu.my, hafizil@psmza.edu.my, shafiq.suhaimi@gmail.com). experimental results. From these empirical correlations, some of the models have been developed by solving coupled of equations between the potential and space charge by finite differences method [5].

Due to complexity of the geometry, some of the researcher only focused on 2D of ESP models [6]-[8]. Some of them only assumed an ideal (uniform) velocity distribution at the inlet boundary condition but in the real case, the velocity should be considered as a real velocity distribution at inlet boundary.

In this work, the description of ESP operation is based on simulation analysis whereby focused on the gas flow rate inside the ESP by implementing five various velocity at the inlet. In this study, the gas flow through the ESP is modeled numerically using computational fluid dynamics (CFD) in order to give insight to the flow behavior inside the ESP. Thus, the model was created using the commercially available CFD code Fluent by Ansys.

\section{Procedure For PAPER SUbMission}

The geometrical details of the ESP are presented in the Fig. 1. The small scale of ESP is being used in the diesel engine in order to trap the particulate matter (PM). The dimension of the small scale ESP model is based on the calculation in order to get more than $90 \%$ of the efficiency. The model consisted of a rectangular collection chamber. The effective length, width and height of the ESP are $1924 \mathrm{~mm}, 352 \mathrm{~mm}$ and 452 $\mathrm{mm}$, respectively. There are seven movable collection plates with $1 \mathrm{~mm}$ thickness were placed inside the ESP chamber.

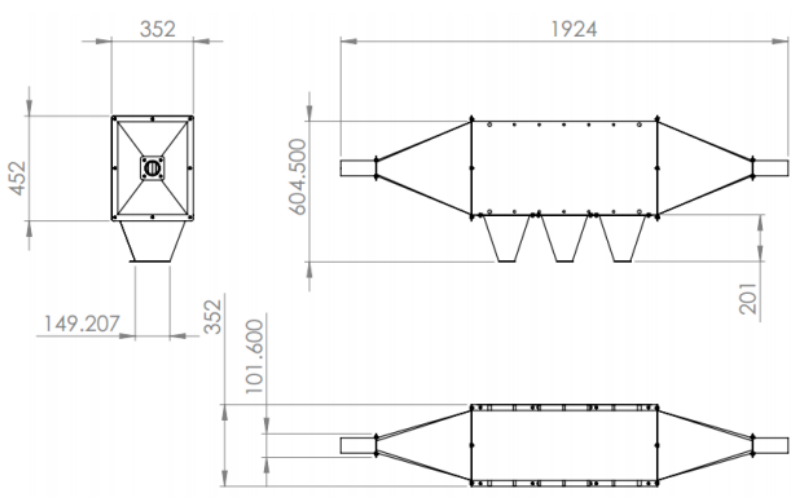

Fig. 1. Schematic diagram of ESP

\section{NUMERICAL APPROACH AND SIMULATION ProcEDURE}

In the numerical computation of fluid transport includes conservation of mass, momentum and turbulence model 
equations

\section{A. Governing Equations}

The air inside the ESP was taking into account the assumption of an incompressible Newtonian fluid due to the small pressure drop occurs across it. The flow was assumed to be steady and can be described by the conservation of mass equation:

$$
\frac{\partial \rho}{\partial \mathrm{t}}+\vec{\nabla} \cdot(\rho \overrightarrow{\mathrm{U}})=0
$$

and the momentum equation:

$$
\begin{aligned}
& \frac{\partial \vec{U}}{\partial \mathrm{t}}+\overrightarrow{\mathrm{U}} \cdot \vec{\nabla} \overrightarrow{\mathrm{U}}=-\frac{\overrightarrow{\nabla \mathrm{p}}}{\rho}+v \vec{\nabla}^{2} \overrightarrow{\mathrm{U}}+\vec{g} \frac{\partial \rho}{\partial \mathrm{t}}+ \\
& \vec{\nabla} \cdot(\rho \overrightarrow{\mathrm{U}})=0
\end{aligned}
$$

For the turbulent flow inside the ESP, the accurate description of the turbulent behavior of the flow is the key of the success of CFD. There are many of turbulent models available in Fluent such as Reynolds stress model, $k-\varepsilon$ model, $k$ - $\omega$ model and etc. In this study, $k$ - $\varepsilon$ model is used due to this model is a recent development and contains a new formulation for the turbulent viscosity and a new transport equation for the dissipation rate, $\varepsilon$. The governing equation of $\mathrm{k}-\varepsilon$ model is shown below:

$$
\begin{gathered}
\frac{\partial}{\partial t}(\rho k)+\frac{\partial}{\partial x_{j}}\left(\rho k u_{j}\right)=\frac{\partial}{\partial x_{j}}\left[\left(\mu+\frac{\mu_{t}}{\sigma_{k}}\right) \frac{\partial k}{\partial x_{j}}\right]+ \\
G_{k}+G_{b}+\rho \varepsilon-Y_{M}+S_{k} \\
\frac{\partial}{\partial t}(\rho \varepsilon)+\frac{\partial}{\partial x_{j}}\left(\rho \varepsilon u_{j}\right)=\frac{\partial}{\partial x_{j}}\left[\left(\mu+\frac{\mu_{t}}{\sigma_{k}}\right) \frac{\partial \varepsilon}{\partial x_{j}}\right]+ \\
\rho C_{1} S_{\varepsilon}-\rho C_{0} \frac{\varepsilon^{2}}{k+\sqrt{v \varepsilon}}+C_{1 \varepsilon} \frac{\varepsilon}{k} C_{3 \varepsilon} G_{b}+S_{\varepsilon}
\end{gathered}
$$

where,

$$
C_{1}=\max \left[0.43, \frac{\eta}{\eta+5}\right], \eta=S \frac{k}{\varepsilon}, S=\sqrt{2 S_{i j} S_{i j}}
$$

The turbulence intensity can be estimated from the following formula derived from an empirical correlation for flow pipe, which is defined as the ratio of the root-mean-square of the velocity fluctuation to the mean flow velocity.

$$
I=\frac{u^{\prime}}{u_{\text {avg }}}=0.16\left(R e_{D_{H}}\right)^{-1 / 8}
$$

The turbulent kinetic energy $k$ and turbulent dissipation rate $\varepsilon$ were calculated using the relations below:

$$
\begin{gathered}
k=\frac{3}{2}\left(u_{\text {avg }} I\right)^{2} \\
\varepsilon=C_{\mu}^{3 / 4} \frac{k^{3 / 2}}{l}
\end{gathered}
$$

where $C_{\mu}$ is an empirical constant specified in the turbulence model (approximately 0.09) and $l$ is the turbulence length scale coming from the next relation:

$$
I=0.07 D_{H}
$$

To estimate the pressure drop across the perforated plates, a source term was added to the $k$ - $\varepsilon$ turbulence model equation. The perforated plates are modeled as a thin porous media of finite thickness with directional permeability over which the pressure change is defined as a combination of viscous loss term and an inertial loss term and given by:

$$
\Delta p=-\left(\frac{\mu}{\alpha} U+C_{2} \frac{1}{2} \rho U^{2}\right) \Delta m
$$

where $\mu$ is the laminar fluid viscosity, $\alpha$ is the permeability of the plate, $C_{2}$ is the pressure loss coefficient per unit thickness of the plate, $v$ is the velocity normal to the porous face and $\Delta m$ is the thickness of the plate. From the study conducted by Haque et al. [9], the pressure drop across the perforated plate is mainly due to the inertial loss at turbulence flow condition. Thus, the viscous loss term was considered negligible and can be eliminated from Eq. (8).

\section{B. Boundary Conditions}

In order to ease the complexity of the geometry, 2D geometry model was considered for the simulation purposed. The developed model was refined using grid adaption utility of Fluent in order to increase the number of grid elements of the computational. The finite volume method was used to discretized the partial differential equations of the model using the SIMPLEC method for pressure-velocity coupling and the first order and second order upwind scheme in order to interpolate the variables on the surface of the control volume. Five simulations were performed with different inlet velocity starting from $10 \mathrm{~m} / \mathrm{s}$ until $50 \mathrm{~m} / \mathrm{s}$ and all are assumed with the uniform velocity distribution. The turbulence of the flow was calculated using turbulent kinetic energy $(k)$ and turbulent dissipation rate $(\varepsilon)$ using given above. A converged solution was used in the simulation after approximately 300 iteration for all the velocity.

\section{RESULTS AND DisCUSSIONS}

\section{A. Figures and Tables}

The velocity vector at $10 \mathrm{~m} / \mathrm{s}$ of the inlet boundary velocity using uniform velocity is presented in Fig. 2. Based on the Fig. 2, the highest velocity was observed at the center of the inlet and at the bottom of the ESP chamber. Only one of the 
plate-to plate has a lower velocity inside the ESP chamber.

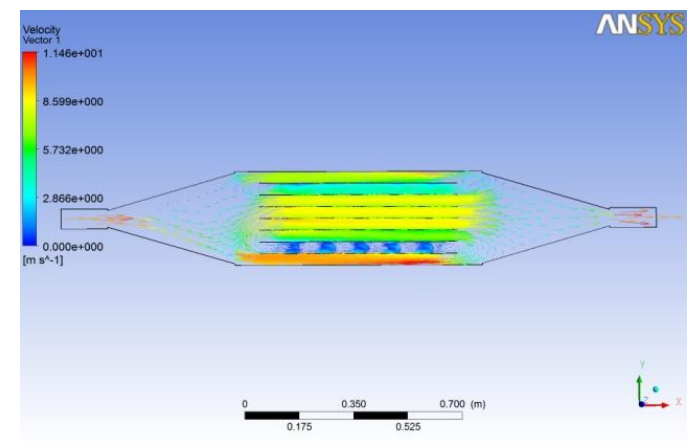

Fig. 2. Velocity vector at $10 \mathrm{~m} / \mathrm{s}$ at inlet

For the velocity vector at $20 \mathrm{~m} / \mathrm{s}$, the flow enters the ESP chamber is not smooth as the three of the plate-to-plate has a lower velocity due to the speed of the flow is increased. As the velocity is increased at $30 \mathrm{~m} / \mathrm{s}$, shown in Fig. 4, the flow has the highest velocity at the bottom of the ESP chamber. As the velocity is increased, it can be seen the turbulent flow is occurred at the top of the ESP chamber.

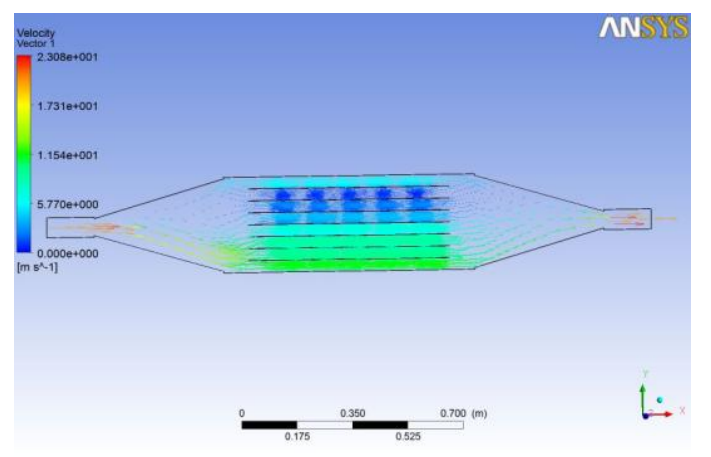

Fig. 3. Velocity vector at $20 \mathrm{~m} / \mathrm{s}$ at inlet

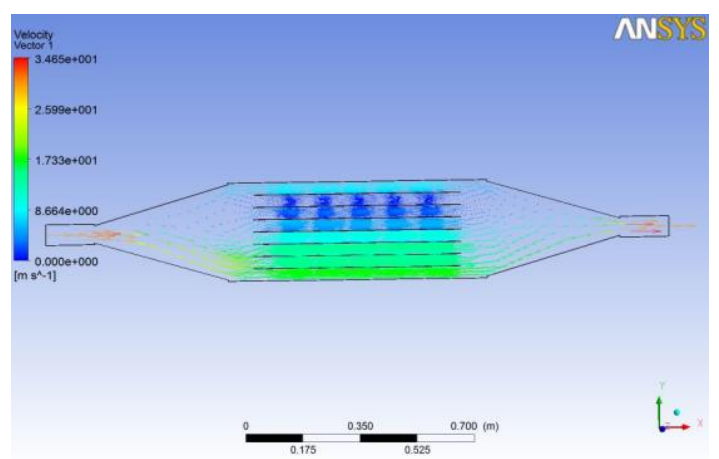

Fig. 4. Velocity vector at $30 \mathrm{~m} / \mathrm{s}$ at inlet

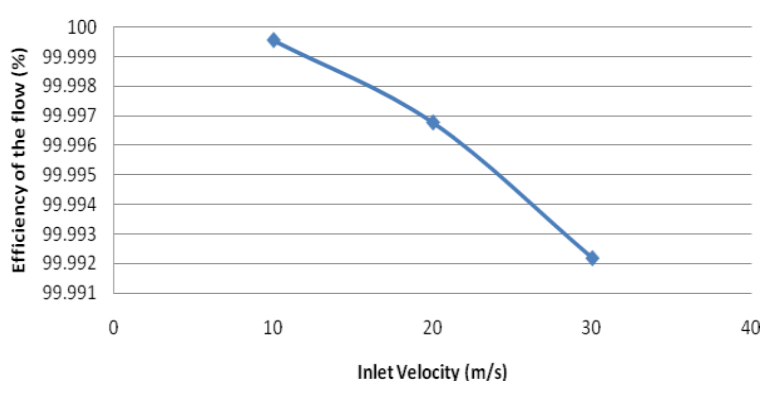

Fig. 5. Efficiency of the flow between inlet and outlet

Even though there is turbulence at higher velocity is observed, the efficiency is still can be consider as high which is more than $99 \%$ of the efficiency of the velocity speed between the inlet and outlet. Thus, ESP is suitable to be used in the diesel engine due to higher efficiency of the flow.

\section{CONCLUSION}

A fluid flow model of ESP is developed using CFD code FLUENT. Thus, realizable $k-\varepsilon$ model was used to compute the turbulence parameters inside the ESP. Simulation were performed with three velocity profiles of uniform flow. From the results obtained, it is found that, ESP has high flow efficiency. Overall, all the three velocities give more than $90 \%$ of efficiency of flow using ESP. Thus, it will have lower pressure drop and suitable to be installed in the diesel engine to increased the performance of the diesel engine.

\section{ACKNOWLEDGMENT}

Universiti Malaysia Pahang is greatly acknowledged for the technical and financial supports under UMP Short grant (RDU110112).

\section{REFERENCES}

[1] S. Kim and K. Lee, "Experimental study of electrostatic precipitator performance and comparison with existing theoretical prediction models," Journal of Electrostatics, vol. 48, pp. 3-25, 1999.

[2] Y. Zhuang et al., "Experimental and theoretical studies of ultra-fine particle behavior in electrostatic precipitators," Journal of Electrostatics, vol. 48, pp. 245-260, 2000.

[3] J. Bapat, "Application of ESP for gas cleaning in cement industry-with reference to India," Journal of Hazardous Materials, vol. 81, pp. 285-308, 2001.

[4] S. Kim et al., "Theoretical model of electrostatic precipitator performance for collecting polydisperse particles," Journal of Electrostatics, vol. 50, pp. 177-190, 2001.

[5] E. Lami et al., "A mathematical model of electrostatic field in wires-plate electrostatic precipitators," Journal of Electrostatics, vol. 39, pp. 1-21, 1997.

[6] L. Zhao et al., "A numerical model of a wire-plate electrostatic precipitator under electrohydrodynamic flow conditions," in Proc. of 10th Int. Conf. on Electrostatic Precipitation ICESP X, 2006, pp. 25-29.

[7] G. Skodras et al., "Particulate removal via electrostatic precipitators - CFD simulation," Fuel Processing Technology, vol. 87, pp. 623-631, 2006.

[8] S. J. Park and S. S. Kim, "Electrohydrodynamic flow and particle transport mechanism in electrostatic precipitators with cavity walls," Aerosol Science \& Technology, vol. 33, pp. 205-221, 2000.

[9] S. Haque et al., "The influence of flow distribution on the performance improvement of electrostatic precipitator," in $C D-R O M$ proceedings of the international conference on air pollution abatement technologies-Future challenges, Cairns, Australia, 2006.

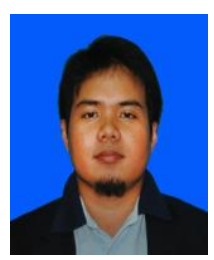

A. F. Yusop was born in Kuala Lumpur, Malaysia on 25 January 1988. After finished his high school, he was obtained a degree in Aeronautical Engineering in Universiti Teknologi Malaysia located in Malaysia on 2010. He became a member of Board of Engineer Malaysia (BEM) on 2010.

Previously, he work as an engineer at JK Wire Sdn. Bhd at Johor, Malaysia. Now currently, he was furthering my studies under the SLAB scholarship or known as fellowship scheme in Phd. $\mathrm{He}$ is doing the particulate matter reduction using electrostatic precipitator (ESP) in diesel engine

Mr. A.F. Yusop is a member of professional body \& professional experience of the Institution of Engineers, Malaysia (IEM No. 40349), Certified ISO 9001:2008 and TS 16949 Automotive Process Approach Auditing and Occupational Safety and Health Course (OSHA). He obtained a bronze medal in Creation, Innovation, Technology \& Research Exposition (CITREX 2013) with the tittle of the project "Particulate Matter (PM) Reduction Technology in Diesel Engine using Electrostatic Precipitator (ESP)". 